\title{
Efficient Gene Knockout in Salivary Gland Epithelial Organoid Cultures
}

Rei Sekiguchi ${ }^{1}$, Madison M. Mehlferber ${ }^{1,2}$, Kazue Matsumoto ${ }^{1}$ and Shaohe Wang ${ }^{1}$

${ }^{1}$ Cell Biology Section, National Institute of Dental and Craniofacial Research, National Institutes of Health, Bethesda, MD 20892, USA

${ }^{2}$ Present address: Department of Biochemistry and Molecular Genetics, University of Virginia, Charlottesville, VA 22903, USA.

Correspondence to: Shaohe Wang (shaohe.wang@nih.gov)

\section{Keywords:}

CRISPR, Cas9, lentivirus, HSP47, integrin, morphogenesis

\section{One-sentence description of our article:}

We present a CRISPR-Cas9 mediated gene perturbation system in mouse embryonic salivary epithelial organoid cultures and use it to demonstrate a critical role for $\beta 1$ integrin in branching morphogenesis. 


\section{ABSTRACT}

We have developed methods to achieve efficient CRISPR-Cas9 mediated gene knockout in ex vivo mouse embryonic salivary epithelial organoids. Salivary epithelial organoids provide a valuable model for characterizing cell signaling, differentiation, and epithelial morphogenesis, but research has been limited by a paucity of efficient gene perturbation methods. Here, we demonstrate highly efficient gene perturbation by transient transduction of guide RNA-expressing lentiviruses into Cas9-expressing salivary epithelial buds isolated from Cas9 transgenic mice. We first show that salivary epithelial buds can be cultured in lowconcentration, non-solidified Matrigel suspensions in 96-well plates, which greatly increases sample throughput compared to conventional cultures embedded in solidified Matrigel. We then describe an efficient workflow to produce experiment-ready, high-titer lentiviruses within 1 week after molecular cloning. To track transduced cells, we designed the lentiviral vector to coexpress a nuclear fluorescent reporter with the guide RNA. We routinely achieve $50-80 \%$ transduction efficiency as indicated by the fluorescent reporter. Importantly, we detected robust loss of targeted protein products when testing 8 guide RNAs for 3 different genes. Moreover, targeting the $\beta 1$ integrin gene (Itgb1) inhibited branching morphogenesis, which supports the importance of cell-matrix adhesion in driving branching morphogenesis. In summary, we have established a lentivirus-based method that can efficiently perturb genes of interest in salivary epithelial organoids, which will greatly facilitate studies of specific gene functions using this system. 


\section{INTRODUCTION}

The mouse salivary gland is an excellent model for studying mammalian organ development. During mouse embryogenesis, an epithelial bud bulges from the oral epidermis to become surrounded by neural crest-derived mesenchyme to form the primordial salivary gland (Jaskoll et al. 2001). Between the epithelium and mesenchyme is a dense, thin layer of extracellular matrix, the basement membrane (Sekiguchi and Yamada 2018). The initial epithelial bud grows and repetitively splits into numerous buds arranged in a "bunch of grapes" appearance through branching morphogenesis, while epithelial cells differentiate into ductal, acinar, or myoepithelial cells, whose concerted actions enable saliva secretion in matured organs (Patel et al. 2006; Tucker 2007; Wang et al. 2017).

Salivary gland research has been advanced by a combination of in vivo and ex vivo approaches. Mouse gene knockout studies definitively established the importance of various signaling pathways, transcriptional factors, and extracellular matrix regulation in salivary gland development (Patel et al. 2006; Knosp et al. 2015; Chatzeli et al. 2017; Emmerson et al. 2017; Szymaniak et al. 2017; Athwal et al. 2019). On the other hand, the ex vivo organotypic culture of mouse embryonic salivary glands enabled characterizations and manipulations that are only possible outside embryos (Borghese 1950). For example, live imaging of ex vivo cultured salivary glands revealed extensive dynamics of epithelial cells and the basement membrane matrix, which catalyzed the discovery that a combination of strong cell-matrix and weak cell-cell adhesions in surface epithelial cells drives branching morphogenesis of salivary glands (Larsen et al. 2006; J.C. Hsu et al. 2013; Harunaga et al. 2014; Wang et al. 2021). Furthermore, the mesenchyme encapsulating the salivary epithelium can be surgically removed to allow mesenchyme-free culture of the salivary epithelium in the presence of basement membrane extract and certain growth factors (Nogawa and Takahashi 1991). These salivary epithelial organoids enabled analysis of how different growth factors affect their growth, morphogenesis, or differentiation (Steinberg et al. 2005; Nakao et al. 2017). 
Small-molecule inhibitors, blocking antibodies, soluble dominant-negative receptors, and small interfering RNA (siRNA) have all been used to perturb gene functions in ex vivo cultures of salivary glands (Sakai et al. 2003; Steinberg et al. 2005; J.C. Hsu et al. 2013), although each has some limitations. Small-molecule inhibitors can be effective to perturb certain signaling pathways or enzymatic activities, but their performance varies depending on their efficacy and specificity. Blocking antibodies or soluble dominant-negative receptors can selectively inhibit ligand binding to surface receptors, but they are only available for a small number of targets. While siRNA-mediated gene knockdown can generalize to most genes, it comes with many disadvantages including transient inhibition, unpredictable knockdown levels, and significant offtarget effects.

Compared to siRNA-mediated gene knockdown, CRISPR-Cas9 mediated gene knockout offers persistent disruption and better specificity (Boettcher and McManus 2015). CRISPR-Cas9 is an RNA-guided DNA endonuclease that can make site-specific doublestranded DNA breaks in mammalian cells (Jinek et al. 2012; Cong et al. 2013). These DNA breaks trigger endogenous DNA repair machinery to catalyze non-homologous end joining (NHEJ) or homology-directed repair (HDR), which can be leveraged for various purposes of genome editing. Notably, knockout of protein coding genes can be readily achieved by frameshift mutations introduced by error-prone NHEJ repair. CRISPR-Cas9 has not been used in ex vivo salivary gland cultures, partly due to a lack of an efficient delivery system for the single guide RNA (sgRNA) needed to target a specific gene.

Here, we demonstrate highly efficient CRISPR-Cas9 mediated gene knockout in ex vivo cultures of mouse embryonic salivary epithelial organoids, which was achieved by transient transduction of sgRNA-expressing high-titer lentiviruses into Cas9-expressing salivary epithelial buds isolated from Cas9 transgenic mice (Platt et al. 2014). To facilitate application of this method, we developed new isolation and culture techniques for salivary epithelial organoids that greatly improved sample throughput, and an efficient workflow to produce experiment-ready, 
high-titer lentiviruses within 1 week after molecular cloning. Importantly, we showed that branching morphogenesis was inhibited when the $\beta 1$ integrin gene (Itgb1) was targeted, indicating our lentiviral delivery system is efficient enough to produce loss-of-function phenotypes at the tissue level. This approach has broad applications to test the roles of genes that may contribute to salivary gland development or disease.

\section{MATERIALS AND METHODS}

\section{Mouse strains}

Mouse experiments were approved by the NIDCR Animal Care and Use Committee (Animal Study Protocols 17-845 and 20-1040). Timed pregnant ICR (CD-1) outbred mice were obtained from Envigo to get wildtype embryos. The Cas9 transgenic mice (Platt et al. 2014) were obtained from the Jackson Laboratory (JAX, 026558). To generate embryos at specific gestational stages, transgenic mice 8-16 weeks old were bred, where the next day after a vaginal plug was found was counted as embryonic day 1.

\section{sgRNA design}

The first half of the coding sequence of a target gene was used for sgRNA design using CRISPOR (http://crispor.tefor.net/) (Concordet and Haeussler 2018) with the UCSC mm10 mouse reference genome and the NGG protospacer adjacent motif. Two or three sgRNAs with both high specificity and efficiency scores were selected for each target gene (P.D. Hsu et al. 2013; Bae et al. 2014; Doench et al. 2016; Chen et al. 2019). sgRNAs with motifs predicted to lower gene knockout efficiencies were avoided (Graf et al. 2019). An extra G was added to the 5'-end if the sequence did not begin with $\mathrm{G}$ to facilitate transcription by the U6 promoter.

\section{Plasmids}


The parental lentiviral vector (pW212; Addgene, 170810) co-expressing sgRNA with an red nuclear fluorescent reporter (NLS-mScarlet-I) and a blasticidin resistance gene was previously generated by our group (Wang et al. 2021). To make the parental vector (pW299; Addgene, TBD) with a blue nuclear fluorescent reporter (NLS-tagBFP), mScarlet-I in pW212 was replaced with tagBFP using Gibson Assembly (Gibson et al. 2009). Two synonymous mutations were introduced to tagBFP to remove an Esp3I site. For cloning of lenti-sgRNA plasmids, a pair of oligos with the desired sgRNA sequence (see sgRNA design) plus a 4-bp 5'-extension ("cacc" for the forward oligo and "aaac" for the reverse complementary oligo) were annealed, and the resulting oligo duplex and lentiviral vectors (pW212 or pW299) digested by Esp3I (NEB, R0734S) were ligated using a 1:2 mixture of T4 ligase (NEB, M0202L) and T4 polynucleotide kinase (NEB, M0236L) in T4 ligase buffer. The ligation mix was transformed using NEB stable competent cells (NEB, C3040). Correct insertion of sgRNA sequence was confirmed by Sanger sequencing.

\section{Lentivirus packaging}

Lentivirus packaging was performed as previously described (Wang et al. 2021). Briefly, lenti-sgRNA plasmids were co-transfected with psPAX2 (Addgene, 12260) and pMD2.G (Addgene, 12259) into HEK293T cells by calcium co-precipitation to produce infectious lentiviral particles. Pooled lentivirus-containing media collected at 36- and 60-hours post transfection were passed through a $0.45 \mu \mathrm{m}$ filter (MilliporeSigma, SE1M003M00), and concentrated using PEG (System Biosciences, LV825A-1) following the manufacturer's instructions. Virus titer was estimated using Lenti-X GoStix Plus (Takara, 631281) after 100x dilution following the manufacturer's instructions. Concentrated lentiviruses were stored at $-80^{\circ} \mathrm{C}$.

\section{Salivary epithelial bud isolation}


Mouse submandibular salivary glands were isolated from 13 or 13.5 day embryos as previously described (Sequeira et al. 2013). The attached sublingual gland was removed by dissection after each gland was isolated to ensure all epithelial buds were from submandibular glands. Glands were treated with 2 units/mL dispase (Thermo Fisher, 17105041; diluted in DMEM/F-12) in a Pyrex spot plate (Fisher Scientific 13-748B; 6-10 glands per well) for 15 min at $37^{\circ} \mathrm{C}$. To quench dispase activity, glands were washed twice with $5 \%$ BSA (MilliporeSigma, A8577; diluted in DMEM/F-12). While being monitored under a dissecting microscope, glands were repetitively triturated using a $200 \mu \mathrm{L}$ pipettor set at $100 \mu \mathrm{L}$ with a low-retention tip (Rainin, 30389187), until the mesenchyme was dissociated into single cells while epithelial buds remained intact. Salivary epithelial buds were rinsed 3 times by being transferred to new wells of the spot plate prefilled with $150 \mu \mathrm{L} 5 \%$ BSA in DMEM/F-12 using a $20 \mu \mathrm{L}$ pipettor with a lowretention pipette tip (Rainin, 30389190). Care was taken during transfer to minimize carryover of mesenchymal cells. To prevent evaporation, the well was covered with a glass coverslip during preparation of lentivirus transduction (see Lentivirus transduction of salivary epithelial buds) and organoid culture media (see Organoid culture). Before the next steps, epithelial buds were further rinsed twice in DMEM/F-12 without BSA.

\section{Lentivirus transduction of salivary epithelial buds}

Lentivirus transduction was performed in the wells of an ultra-low attachment 96-well Vbottom plate (S-bio, MS-9096VZ). Each epithelial bud was transferred into one well in precisely $5 \mu \mathrm{L}$ medium. A $15 \mu \mathrm{L}$ lentivirus treatment mixture containing $10 \mu \mathrm{L}$ lentivirus stock, $4 \mu \mathrm{L}$ DMEM/F-12 and $1 \mu \mathrm{L} 160 \mu \mathrm{g} / \mathrm{mL}$ polybrene (MilliporeSigma, H9268) was added to each well. The plate was incubated in a humidified $37^{\circ} \mathrm{C}$ incubator for $1-2$ hours. Each bud was washed 3 times in DMEM/F-12 before culture (see Organoid culture).

\section{Organoid culture}


Before salivary gland dissections, aliquots of growth factor-reduced Matrigel (Corning, 356231 ; stock $9-10 \mathrm{mg} / \mathrm{mL}$ ) were thawed at $4^{\circ} \mathrm{C}$ or on ice. The volume was calculated as $5 \mu \mathrm{L}$ per organoid with 1 or $2 \mu \mathrm{L}$ extra to compensate for pipetting errors. The base medium for culture was DMEM/F-12 (Thermo Fisher, 11039047) supplemented with 1x PenStrep (100 units/mL penicillin, $100 \mu \mathrm{g} / \mathrm{mL}$ streptomycin; Thermo Fisher, 15140163), hereafter referred to as DMEM/F-12-PS.

Epithelial buds were cultured in ultra-low attachment 96-well V-bottom plates (S-bio, MS9096VZ) as previously described (Wang et al. 2021). Briefly, one bud was cultured in each well in the organoid culture media with $0.5 \mathrm{mg} / \mathrm{mL}$ growth factor-reduced Matrigel (Corning, 356231; stock 9-10 mg/mL), $200 \mathrm{ng} / \mathrm{mL}$ FGF7 (R\&D Systems, 5028-KG-025), and 1x ITS supplement (10 mg/L insulin, $5.5 \mathrm{mg} / \mathrm{L}$ transferrin, $6.7 \mu \mathrm{g} / \mathrm{L}$ selenium; Thermo Fisher, 41400045) in DMEM/F-12-PS. 10 ng/mL NRG1 (R\&D Systems, 9875-NR-050) was included in some testing culture conditions. In practice, the organoid culture media was prepared at $2 x$ concentration with a total volume of $(n+2) \times 50 \mu \mathrm{L}$, where $\mathrm{n}$ is the sample number. The wells for organoid culture were pre-filled with $47 \mu \mathrm{L}$ DMEM/F-12-PS, and one bud was transferred into each well in precisely $3 \mu \mathrm{L}$ medium using a low-retention pipette tip. $50 \mu \mathrm{L} 2 \mathrm{x}$ organoid culture media was then added to each well. The plate was cultured at $37^{\circ} \mathrm{C}$ with $5 \% \mathrm{CO}_{2}$.

\section{Immunostaining of organoids}

Immunostaining of organoids was performed as previously described (Wang et al. 2021). Briefly, organoids were fixed in 4\% PFA (Electron Microscopy Sciences, 15710) in PBS for 1 hour at room temperature (RT) or overnight at $4^{\circ} \mathrm{C}$, permeabilized in PBSTx (PBS with $0.2 \%$ Triton-X-100; Thermo Fisher, 28314) for $30 \mathrm{~min}$ at RT, blocked in 5\% donkey serum (Jackson ImmunoResearch, 017-000-121) in PBSTx for 2 hours at RT, incubated in primary antibodies diluted in 5\% donkey serum in PBSTx for 2 days at $4^{\circ} \mathrm{C}$, washed $4 \times 15$ min each in PBSTx at RT, incubated in secondary antibodies diluted in PBSTx for 2 days at $4^{\circ} \mathrm{C}$, washed $4 \times 15$ min in 
PBSTx at RT, rinsed once in PBS, and mounted in antifade mountant (Thermo Fisher, P36930) supported by two layers of imaging spacers (Grace Bio-labs, 654004). Immunostaining of $\beta 1$ integrin used Atto-565-labeled primary antibodies with 7-day incubation at $4^{\circ} \mathrm{C}$ without secondary antibody staining. All incubation was performed in sample baskets (Intavis, 12.440) in a 24-well plate.

The following antibodies and concentrations were used for immunostaining: anti-HSP47 (MilliporeSigma, HPA029198), $1 \mu \mathrm{g} / \mathrm{mL}$; anti-a9 integrin (R\&D Systems, AF3827-SP), 1 mg/mL; Atto-565-labeled Hamster anti- $\beta 1$ integrin (clone Ha2/5, BD Biosciences 555002), $10 \mu \mathrm{g} / \mathrm{mL}$. Alexa Fluor 647-labeled donkey anti-rabbit or donkey anti-goat secondary antibodies (Jackson ImmunoResearch) were used at 1:200 (1.5-3 $\mu \mathrm{g} / \mathrm{mL})$.

\section{Microscopy}

Phase contrast and epifluorescence images were acquired using a Nikon 10x, 0.3 NA, Plan Fluor objective on a Nikon Ti-E brightfield microscope system with a Hamamatsu Orca Flash 4.0 V3 sCMOS camera controlled by Nikon NIS-Elements software. The JOBS module of the software was used to automatically go through multiple wells in a 96-well plate.

Immunofluorescence images were acquired by laser scanning confocal microscopy using Nikon 20x, 0.75 NA or 40x, 1.3 NA Plan Fluor objectives on a Nikon A1R Confocal Microscope System controlled by Nikon NIS-Elements software, or a Zeiss 63x, 1.4 NA Plan Apo objective on a Zeiss LSM 880 system controlled by Zeiss ZEN software.

\section{Image analysis and quantification}

Image analysis and quantification were performed in Fiji (Schindelin et al. 2012). Customized ImageJ Macro and Python scripts were used for automating or facilitating image analysis and data visualization (see Data, Code and Resource Availability). Before plotting of 
fluorescence intensity, all raw intensity values were normalized to the average intensity of the control group.

The HSP47 intensity was quantified as the mean intensity in the epithelial bud subtracting the background intensity inside the nuclei of the interior bud. The epithelial bud region of interest $(\mathrm{ROI})$ was segmented using the EGFP channel, which was smoothened with a Gaussian filter ( $r=5$ pixels) and binarized using the "Huang" thresholding algorithm. The nuclear ROI was segmented using the DAPI channel, which was smoothened with a Gaussian filter ( $r=1$ pixels), background subtracted with a rolling ball method ( $r=50$ pixels), and binarized using the default thresholding algorithm. The interior bud ROI was obtained by intersecting the nuclear ROI with the epithelial bud ROI shrunken by 20 microns. For each imaging stack, the average measurement of all $z$ slices was calculated as the final HSP47 intensity.

The $\alpha 9$ and $\beta 1$ integrin intensities were quantified as the peak intensity along a $3 \mu \mathrm{m}$ width line across the edge of two transduced cells, subtracting background intensity that is the average intensity of the beginning $10 \%$ of the line length. Five edges were measured on each image from 3-7 organoids per experimental group.

Manual counting of bud number was performed on phase contrast images. File names were scrambled before counting for observer blinding. R.S. performed all counting to avoid between-person variation. S.W. decoded and plotted the counting results.

\section{Data, Code and Resource Availability}

All data of this study are available in Figshare (link to update upon publication). All plasmids are available in Addgene (link to update upon publication). Customized scripts and usage instructions are available from GitHub (link to update upon publication). Selected step-bystep protocols can be found at https://snownontrace.github.io/ . 


\section{RESULTS}

\section{Salivary epithelial bud isolation and organoid culture in 96-well plates}

We first optimized the isolation and culture techniques for salivary epithelial organoids to increase sample throughput (Fig. 1A). Conventionally, salivary gland mesenchyme is removed from the epithelium by dissection after dispase treatment. The whole epithelial rudiment or individual epithelial buds are then embedded in high-concentration Matrigel (a basement membrane extract) that quickly solidified. In our new procedure, multiple salivary glands were triturated in bulk after dispase treatment to dissociate the mesenchyme into single cells, whereas the epithelial buds remained intact, presumably due to stronger cell-cell adhesion. Each epithelial bud was then cultured in non-solidified Matrigel in the well of an ultra-lowattachment 96-well plate, which greatly facilitated sample handling and high-throughput imaging. The new isolation procedure is also much less labor-intensive. For comparison, removing the mesenchyme of 20 glands would require 1-2 hours of careful dissection work using the conventional procedure, but only about 10 minutes using our new procedure.

Next, we tested several combinations of growth factors using the new culture format. We found that epithelial organoids grew best with FGF7, NRG1 and ITS (insulin, transferrin, and selenium; Fig. 1B-C). The organoids failed to grow with only NRG1 and ITS, supporting the critical role of FGF signaling in salivary epithelial development (Steinberg et al. 2005). Removing ITS from the combination significantly slowed organoid growth, whereas removing NRG1 did not affect organoid growth or budding morphogenesis (Fig. 1B-C). Thus, we chose to use the FGF7 and ITS combination for standard organoid cultures.

\section{Streamlined production of high-titer sgRNA-expressing lentiviruses}

To maximize efficiency, we tested the use of lentivirus to express sgRNAs in salivary epithelial organoids. We designed lentiviral vectors to co-express a nuclear fluorescent reporter with the sgRNA (NLS-mScarlet-I or NLS-tagBFP) to follow transduced cells, and a blasticidin 
resistance gene (BlastR) to allow antibiotic selection (Fig. 2A). These vectors also enabled straightforward molecular cloning by one-step ligation of the sgRNA oligo duplex (Fig. 2A), which could be easily adapted for parallel cloning of tens of sgRNA vectors. Using miniprepgrade plasmid DNAs, we generated experiment-ready, high-titer lentiviruses in 5 days (Fig. 2B). To estimate lentivirus titer, we used a quick commercial test to measure the test-band intensity (Fig. 2C) and compared that to a reference virus. The typical titer of sgRNA-expressing lentiviruses using this method was about $1.5 \times 10^{8} \mathrm{IFU} / \mathrm{mL}$. After transient transduction of lentiviruses into epithelial buds, nuclear reporter expression could be detected by the next day and reached a maximum level within two days. We found that $50-80 \%$ of the cells in the organoids expressed nuclear reporters, even without antibiotic selection (Fig. 2D).

\section{Efficient gene perturbation in salivary epithelial organoids}

For gene perturbation, we transduced sgRNA-expressing lentiviruses into Cas9expressing salivary epithelial buds isolated from Cas9 transgenic mice (Platt et al. 2014). We used an sgRNA targeting the $E$. coli lacZ gene as the control and tested 2 or 3 targeting sgRNAs for each of the 3 genes: Serpinh1, Itga9 and Itgb1 (Table 1). We found that all targeting sgRNAs significantly reduced the expression levels of targeted proteins by immunostaining, but different sgRNAs for the same gene could yield different levels of residual proteins (Figs. 3A-D, 4A-B). Interestingly, the efficacy of protein reduction was insensitive to the level of sgRNA expression, because cells with low or high sgRNA reporter expression reduced targeted proteins to similar levels (Fig. 4A, yellow and cyan asterisks). Thus, we conclude that lentivirus-mediated sgRNA delivery can robustly disrupt gene functions, and the efficiency depends on the sgRNA identity.

We next analyzed organoid growth and morphogenesis after gene disruption. Among the 3 targeted genes, Serpinh1 encodes HSP47, a collagen-specific chaperone that is important for collagen biogenesis (Ito and Nagata 2017), whereas Itga9 and Itgb1 encode $\alpha 9$ and $\beta 1$ integrins 
that mediate cell-matrix adhesion (Kechagia et al. 2019). No gross phenotypes were observed when Serpinh1 or Itga9 were disrupted (data not shown). In contrast, the stronger Itgb1 sgRNA (sg2-ltgb1) abolished the normal increase in bud numbers during the third day of culture (Fig. 4C-D). Moreover, when we used antibiotic treatment to select for lentivirus-transduced cells, both sgRNAs of Itgb1 strongly inhibited the increase in numbers of buds (Fig. 4C-D). Therefore, we conclude that $\beta 1$ integrin, but not HSP47 or $\alpha 9$ integrin, is required for branching morphogenesis of salivary epithelial organoids under these culture conditions. Our results provide genetic perturbation evidence that strengthens the finding that $\beta 1$ integrin is required for branching, which was previously established via antibody blocking (J.C. Hsu et al. 2013). We believe the lack of phenotype when targeting Serpinh1 was likely because sufficient collagen was provided by the Matrigel supplement, whereas the lack of phenotype when targeting Itga9 was presumably due to the expression of other a integrins in this tissue that dimerize with $\beta 1$ integrin (e.g., a3, a6 and $a \mathrm{~V})$.

\section{DISCUSSION}

In this study, we describe successful implementation of CRISPR-Cas9 mediated gene knockout in ex vivo salivary epithelial organoid cultures. We achieved robust gene disruption by transient transduction of high-titer sgRNA-expressing lentiviruses into Cas9-expressing salivary epithelial buds isolated from Cas9 transgenic mice. A major advantage of gene ablation in ex vivo cultures is to bypass the need to generate knockout mice to analyze gene functions. To facilitate the application of this method, we established new procedures for isolating and culturing salivary epithelial buds that are both less labor-intensive and higher throughput than conventional procedures. We demonstrated successful protein reduction for 3 targeted genes and inhibited branching morphogenesis when targeting the $\beta 1$ integrin gene. This approach will be broadly useful to analyze the functions of other specific genes using ex vivo cultures of various tissues. 


\section{The efficiency of CRISPR-Cas9 mediated gene disruption}

While siRNA targets the mRNA, CRISPR-Cas9 targets the genomic DNA. The superior efficacy of CRIPR-Cas9 mediated gene disruption is partly because there are usually only 2 copies of genes, whereas the mRNA molecules of the same gene can number tens to thousands. Moreover, DNA disruption is heritable and thus only needs to occur once, whereas siRNAs need to constantly fight against newly transcribed mRNAs, and they become diluted as cells divide.

The efficiency of CRISPR-Cas9 can be optimized by sgRNA design. Mechanistically, gene disruption by CRISPR-Cas9 mainly comes from frameshift mutations introduced during the error-prone NHEJ repair of DNA breaks. Importantly, the error patterns of NHEJ are strongly influenced by the sequence context. Several studies have developed algorithms that predict how likely an sgRNA will cause out-of-frame mutations (Bae et al. 2014; Doench et al. 2016; Chen et al. 2019). These predictive models have been incorporated as a few efficiency and outcome scores into the intuitive web-based sgRNA design tool CRISPOR to guide the choice of sgRNAs (Concordet and Haeussler 2018).

The lentivirus-based sgRNA delivery ensures stable sgRNA expression, which further increases the probability of gene disruption. The double-stranded DNA breaks may be perfectly repaired rarely, but they will again be cut until some errors become incorporated during repair to prevent further cuts by the CRISPR-Cas9 enzyme. It is worth noting that the U6 promoter we use to drive sgRNA expression imposes two additional considerations on the sgRNA design. First, if the sgRNA does not begin with the nucleotide $G$, an extra $G$ should be added to the beginning due to transcriptional start site preference of the U6 promoter (Goomer and Kunkel 1992; Ma et al. 2014). Second, certain sequence motifs at the 3' end of sgRNA can severely lower gene knockout efficiencies (Graf et al. 2019). The sgRNAs containing these motifs are 
highlighted as "inefficient" when using CRISPOR for sgRNA design, and these sgRNAs should be avoided for gene disruption.

\section{The specificity of CRISPR-Cas9 mediated gene disruption}

Both siRNA and CRISPR-Cas9 have off-target effects, but off-targets of CRISPR-Cas9 can be predicted much more reliably and thus be more effectively mitigated with strategic sgRNA design (P.D. Hsu et al. 2013; Cradick et al. 2014; Doench et al. 2016). Cutting by CRISPR-Cas9 requires the protospacer adjacent motif (PAM) and is most sensitive to mismatches within 11 bp of the PAM (Jinek et al. 2012; Cong et al. 2013), but it can tolerate certain insertion/deletion mutations in the sgRNA that causes DNA or RNA bulges (Lin et al. 2014). The PAM for the Streptococcus pyogenes Cas9 (SpCas9) we use is NGG, which is relatively abundant in the coding sequence of genes. As a result, there are often tens to hundreds of sgRNAs with high-specificity scores from which to choose for any target gene. When selecting sgRNAs for gene knockout studies, it is good practice to prioritize high specificity, and to use several sgRNAs for the same target gene.

\section{Applications of CRISPR-Cas9 gene knockout in ex vivo cultures}

Lentivirus-based sgRNA delivery for CRISPR-Cas9 gene knockout in ex vivo cultures can be easily adapted for analyzing gene functions at the tissue or cell level by adjusting lentivirus titers. For tissue-level phenotypes, such as organoid growth and branching morphogenesis, it will be better to use the highest possible titer to transduce most cells in the organoid. The penetrance of perturbation phenotypes may be further enhanced by antibiotic selection of transduced cells. For cell-level phenotypes, however, it will be better to lower the virus titer to generate mosaic knockout, so that only a small fraction of cells is perturbed to maintain the native tissue environment. For example, changes of intracellular organization or transcriptional activities during cell differentiation can be examined by immunostaining or single- 
molecule RNA fluorescence in situ hybridization (smFISH) in mosaic knockout organoids (Raj et al. 2008; Wang 2018).

Our work focuses on epithelial cells, but lentivirus can also transduce many other cell types. One possible future application is to test whether it can mediate efficient gene knockout in mesenchymal cells, which will enable studies of specific gene functions in epithelialmesenchymal interactions.

\section{AUTHOR CONTRIBUTIONS}

R.S. contributed to conception, data acquisition, analysis, drafted and critically revised the manuscript. M.M.M. contributed to data acquisition, and critically revised the manuscript. K.M. contributed to data acquisition, and critically revised the manuscript. S.W. contributed to conception, design, data acquisition, analysis, interpretation, drafted and critically revised the manuscript. All authors gave final approval and agreed to be accountable for all aspects of the work.

\section{ACKNOWLEDGMENTS}

We would like to thank members of the Cell Biology Section for helpful discussions, Kenneth Yamada and Di Wu (NIDDK) for critical reading of the manuscript, the NIDCR Imaging Core for microscopy support, the NIDCR Combined Technical Research Core for Sanger sequencing, and the NIDCR Veterinary Resource Core for animal support. S.W. is supported in part by an NIDCR K99 Pathway to Independence Award (K99 DE27982). This work is supported by the NIH Intramural Research Program (NIDCR, ZIA DE000525). 
FIGURES, TABLES, and FIGURE LEGENDS

A

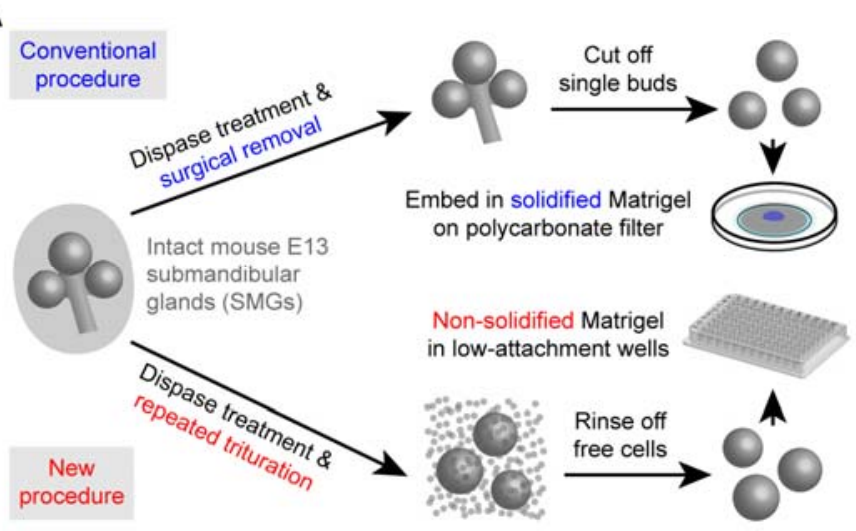

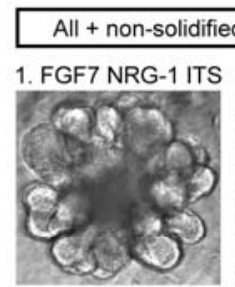

3. FGF7 ITS

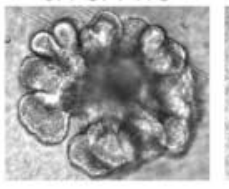

C

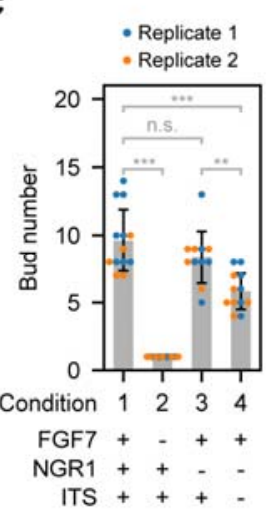

Figure 1. Salivary epithelial bud isolation and organoid culture in 96-well plates. (A)

Schematics showing conventional (upper) and new (lower) procedures to isolate and culture single epithelial buds from mouse E13 submandibular salivary glands. (B) Phase contrast images of single-bud organoid cultures at 48 hours under indicated culture conditions. (C) Plot of bud number per organoid under indicated culture conditions. Error bars, standard deviation. Statistics, Tukey test. ${ }^{* * *}, \mathrm{p}<0.001 .{ }^{* *}, \mathrm{p}<0.01$. n.S., not significant. FGF7, $200 \mathrm{ng} / \mathrm{mL} . \mathrm{NRG} 1,10$ $\mathrm{ng} / \mathrm{mL}$. ITS, $10 \mathrm{mg} / \mathrm{L}$ insulin, $5.5 \mathrm{mg} / \mathrm{L}$ transferrin, and $6.7 \mu \mathrm{g} / \mathrm{L}$ selenium. Non-solidified Matrigel (MG), 400-500 $\mu \mathrm{g} / \mathrm{mL}$. Scale bar, $100 \mu \mathrm{m}$. 
A
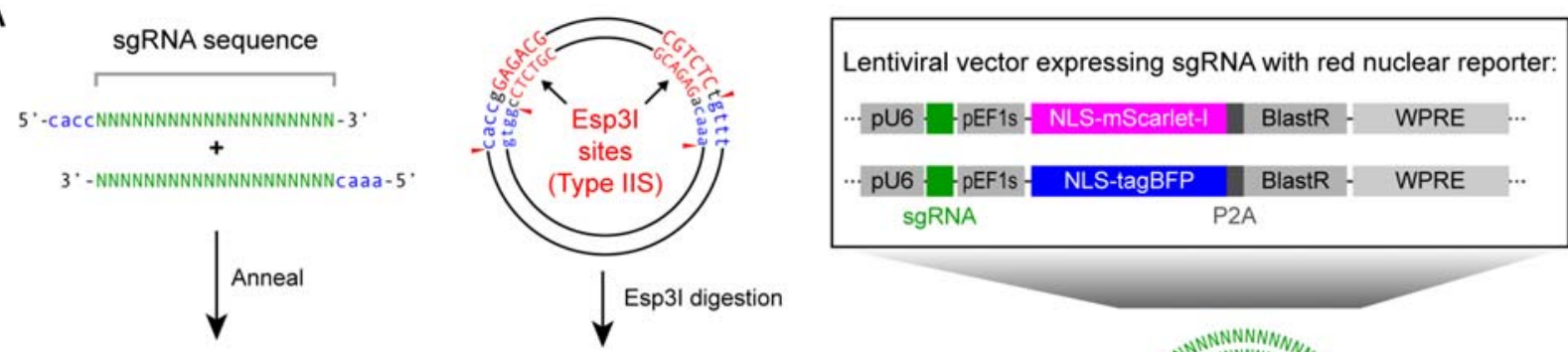

$5^{\circ}$ - caccinnnnnnnnnnnnnnnnnnn $-3^{\circ}$

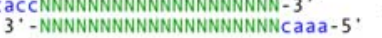

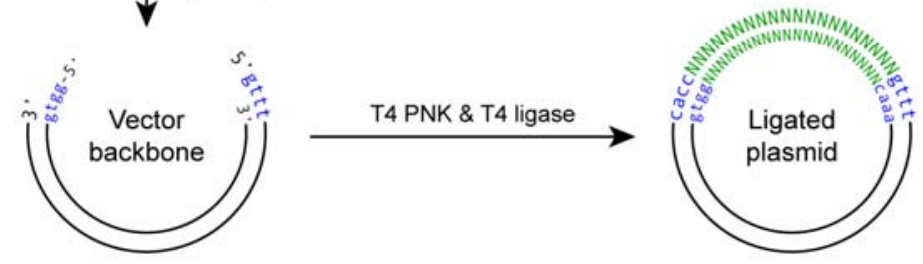

B

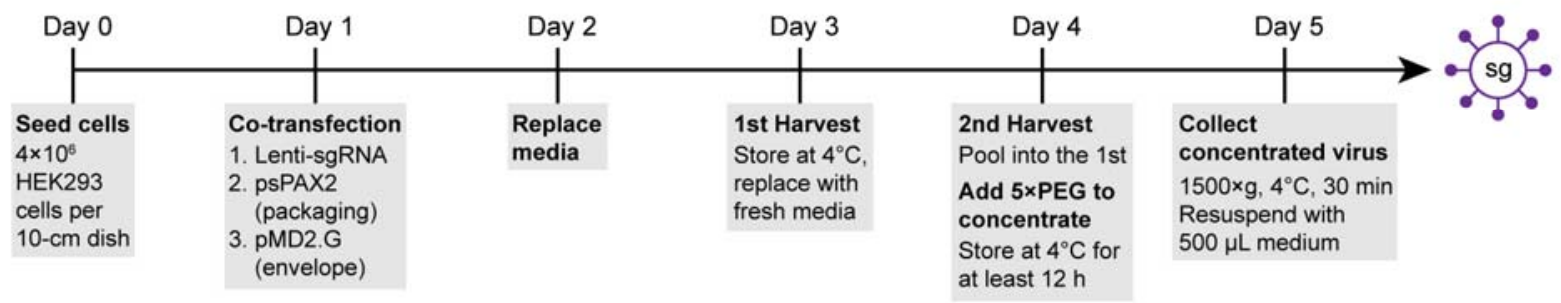

C

Lentivirus titration with Lenti- $X^{\mathrm{TM}}$ GoStix ${ }^{\mathrm{TM}}$ Plus

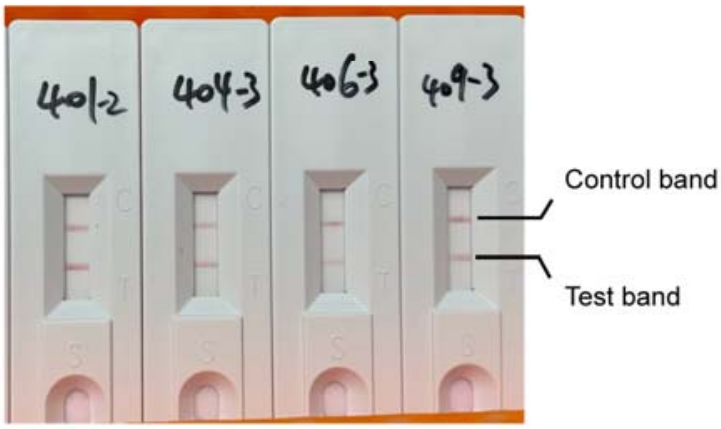

D

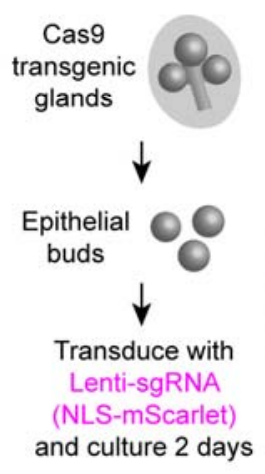

Figure 2. Streamlined production of high-titer sgRNA-expressing lentiviruses. (A)

Schematics showing cloning procedure for the sgRNA-expressing lentiviral vector. (B)

Schematics showing the timeline of lentivirus production. (C) Image of lentivirus titration results using Lenti-X GoStix Plus. (D) Left: schematics of the experimental procedure. Right: maximum intensity projection of confocal fluorescence images of a lentivirus-transduced salivary gland single-bud epithelial organoid. Scale bar, $20 \mu \mathrm{m}$. 
A

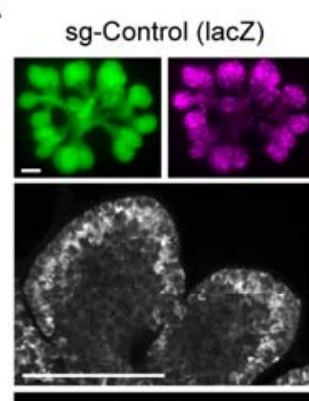

Anti-HSP47 (SERPINH1) NLS-mScarlet-I (sgRNA reporter) EGFP (Cas9 Transgene reporter)

C

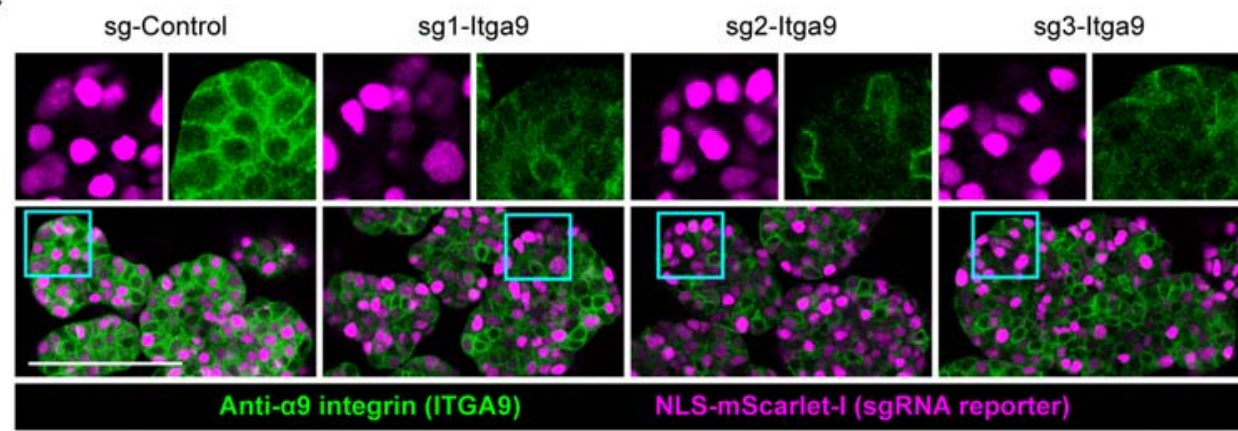

B

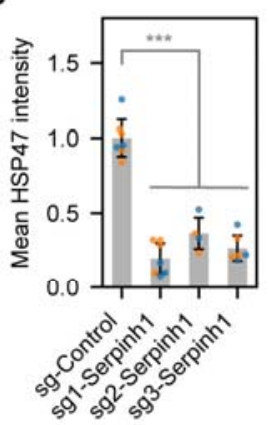

D

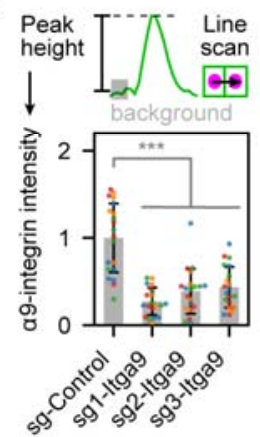

Figure 3. Efficient gene perturbation in Cas9 transgenic salivary gland epithelial

organoids. (A) Widefield (upper) or maximum intensity projection of $10 \mu \mathrm{m} z$-range of confocal (lower) fluorescence images of single-bud organoid cultures transduced with lentiviruses expressing indicated sgRNAs and immunostained with anti-HSP47. EGFP is co-expressed with the Cas9 transgene. The mouse Serpinh1 gene encodes the HSP47 protein. Organoids were fixed at 58 hours post transduction. (B) Plot of mean HSP47 intensity per imaging field ( $\mathrm{n}=2$ organoids per condition). Different colors indicate measurements from different organoids. (C) Confocal fluorescence images of single-bud organoid cultures transduced with lentiviruses expressing indicated sgRNAs and immunostained with anti-a9 integrin. Cyan boxes mark the single-channel magnified images shown above. Organoids were fixed at 72 hours post transduction. (D) Schematic of the quantification method (upper) and plot (lower) of the a9integrin intensity across edges of adjacent transduced cells. Error bars, standard deviation. Statistics, Tukey test. ${ }^{* * *}, p<0.001$. Scale bars, $100 \mu \mathrm{m}$. 
bioRxiv preprint doi: https://doi org/10.1101/2022.02 28.482127; this version posted March 3, 2022. The copyright holder for this preprint (which was not certified by peer review) is the author/funder. This article is a US Government work. It is not subject to copyright under 17 USC 105 and is also made available for use under a CCO license.

A
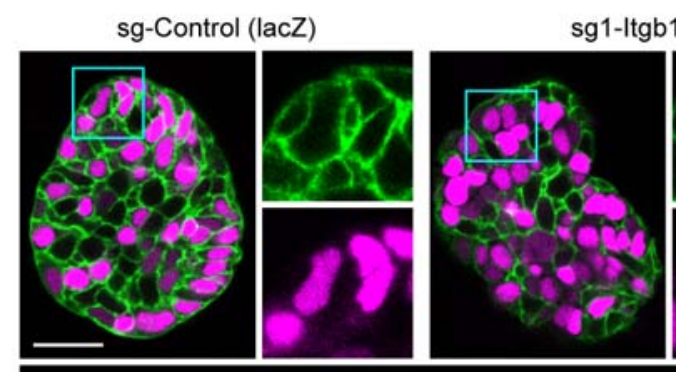

Anti-ß1 integrin (ITGB1)

C

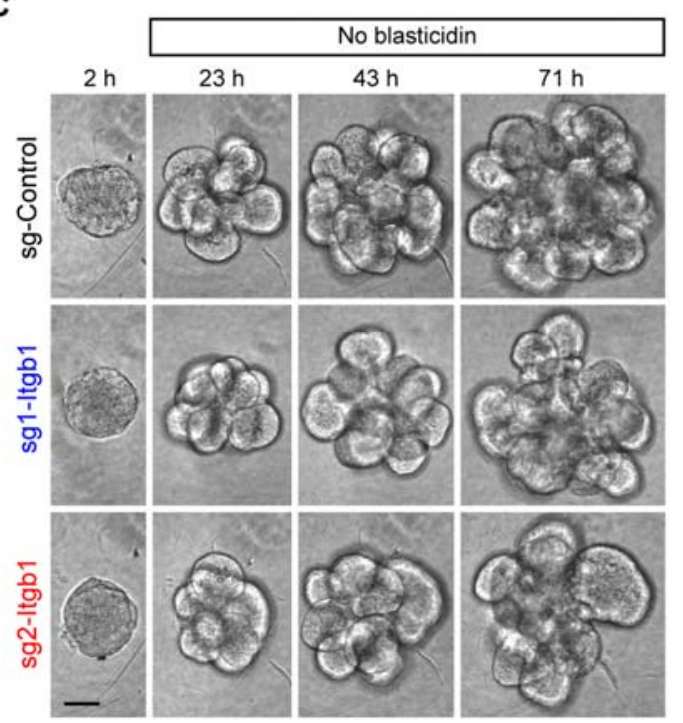

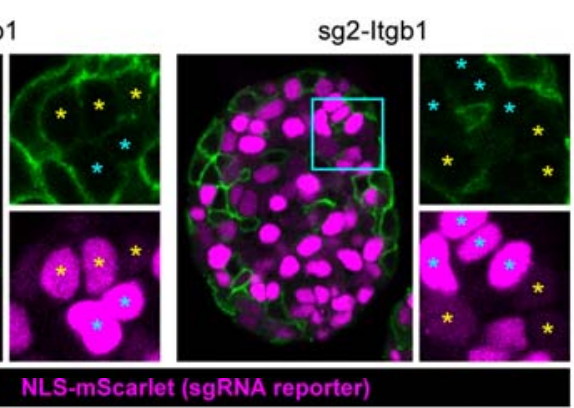

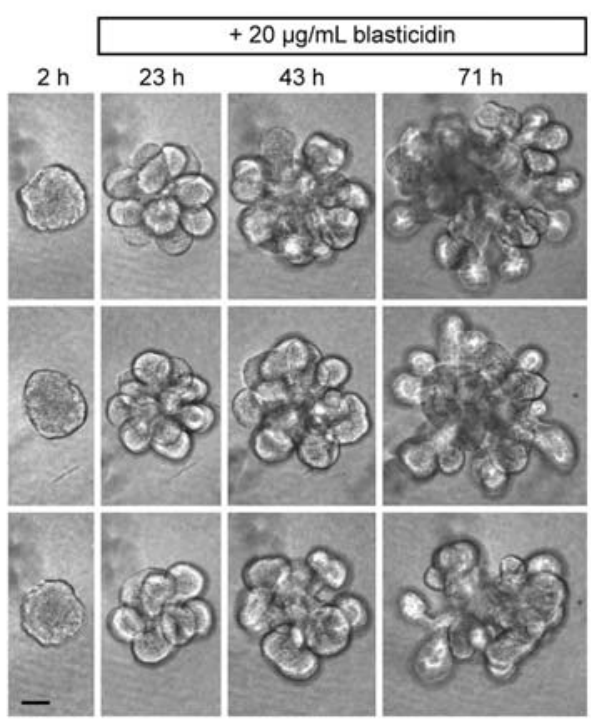

B

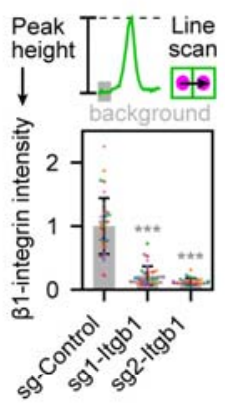

D
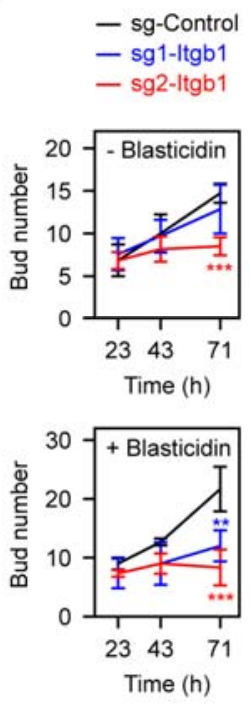

Figure 4. Knockout of $\beta 1$ integrin inhibits budding morphogenesis. (A) Confocal fluorescence images of single-bud organoid cultures transduced with lentiviruses expressing indicated sgRNAs and immunostained with anti- $\beta 1$ integrin. Cyan boxes mark the singlechannel magnified images shown above. Yellow and cyan asterisks mark cells expressing low and high levels of the sgRNA reporter, respectively. Organoids were fixed at 71 hours post transduction. (B) Schematic of the quantification method (upper) and plot (lower) of the $\beta 1$ integrin intensity across edges of adjacent transduced cells. Error bars, standard deviation. (C) Phase contrast images of single-bud organoid cultures transduced with lentiviruses expressing indicated sgRNAs at 4 different time points with or without blasticidin antibiotic selection. (D) Plot of bud number per organoid over time. Statistics, Tukey test. ${ }^{* *}, p<0.01 .{ }^{* * *}, p<0.001$. Scale bars, $20 \mu \mathrm{m}(\mathrm{A}), 100 \mu \mathrm{m}(\mathrm{C})$. 
Table 1. sgRNA sequences used in this study

\begin{tabular}{|l|l|l|l|}
\hline Target gene & sgRNA ID & sgRNA sequence & Plasmid ID \\
\hline LacZ & sg-Control & TGCGAATACGCCCACGCGAT & pW188 \\
\hline Serpinh1 & sg1-Serpinh1 & TAAAGCCACCACAGCGTCGC & pW228 \\
\hline Serpinh1 & sg2-Serpinh1 & CACCACAGCGTCGCAGGCGA & pW229 \\
\hline Serpinh1 & sg3-Serpinh1 & AAGTTCCAAGGCGACCACAC & pW230 \\
\hline Itga9 & sg1-Itga9 & TGCTGGCGCTGGTGGCCGCG & pW317 \\
\hline Itga9 & sg2-Itga9 & TGGGCGGCCCGGCTGCGGCG & pW318 \\
\hline Itga9 & sg3-Itga9 & CCAGCGCCAGCAGCAGCGCG & pW319 \\
\hline Itgb1 & sg1-Itgb1 & CAAACTGATCAATCCAATCCAGG & pW401 \\
\hline Itgb1 & sg2-Itgb1 & GCGGAGAATGTATACAAGCAGGG & pW402 \\
\hline
\end{tabular}




\section{REFERENCES}

Athwal HK, Murphy G, Tibbs E, Cornett A, Hill E, Yeoh K, Berenstein E, Hoffman MP, Lombaert IMA. 2019. Sox10 Regulates Plasticity of Epithelial Progenitors toward Secretory Units of Exocrine Glands. Stem Cell Reports. 12(2):366-380.

doi:10.1016/j.stemcr.2019.01.002. https://doi.org/10.1016/j.stemcr.2019.01.002.

Bae S, Kweon J, Kim HS, Kim JS. 2014. Microhomology-based choice of Cas9 nuclease target sites. Nat Methods. 11(7):705-706. doi:10.1038/nmeth.3015.

Boettcher M, McManus MT. 2015. Choosing the Right Tool for the Job: RNAi, TALEN, or CRISPR. Mol Cell. 58(4):575-585. doi:10.1016/j.molcel.2015.04.028. http://dx.doi.org/10.1016/j.molcel.2015.04.028.

Borghese E. 1950. The development in vitro of the submandibular and sublingual glands of Mus musculus. J Anat. 84(3):287-302.

http://www.ncbi.nlm.nih.gov/pubmed/15436333\%0Ahttp://www.pubmedcentral.nih.gov/ar ticlerender.fcgi?artid=PMC1273304.

Chatzeli L, Gaete M, Tucker AS. 2017. Fgf10 and Sox9 are essential for the establishment of distal progenitor cells during mouse salivary gland development. Development. 144(12):2294-2305. doi:10.1242/dev.146019.

http://dev.biologists.org/content/early/2017/05/11/dev.146019.long.

Chen W, McKenna A, Schreiber J, Haeussler M, Yin Y, Agarwal V, Noble WS, Shendure J. 2019. Massively parallel profiling and predictive modeling of the outcomes of CRISPR/Cas9-mediated double-strand break repair. Nucleic Acids Res. 47(15):7989_ 8003. doi:10.1093/nar/gkz487.

Concordet JP, Haeussler M. 2018. CRISPOR: Intuitive guide selection for CRISPR/Cas9 genome editing experiments and screens. Nucleic Acids Res. 46(W1):W242-W245. doi:10.1093/nar/gky354.

Cong L, Ran FA, Cox D, Lin S, Barretto R, Habib N, Hsu PD, Wu X, Jiang W, Marraffini L a, et 
al. 2013. Multiplex Genome Engineering Using CRISPR/Cas Systems. Science (80- ). 339(6121):819-823. doi:10.1126/science.1231143. [accessed 2013 Feb 27]. http://www.ncbi.nlm.nih.gov/pubmed/23287718.

Cradick TJ, Qiu P, Lee CM, Fine EJ, Bao G. 2014. COSMID: A web-based tool for identifying and validating CRISPR/Cas off-target sites. Mol Ther - Nucleic Acids. 3(12):e214. doi:10.1038/mtna.2014.64. http://dx.doi.org/10.1038/mtna.2014.64.

Doench JG, Fusi N, Sullender M, Hegde M, Vaimberg EW, Donovan KF, Smith I, Tothova Z, Wilen C, Orchard R, et al. 2016. Optimized sgRNA design to maximize activity and minimize off-target effects of CRISPR-Cas9. Nat Biotechnol. 34(2):184-191. doi:10.1038/nbt.3437.

Emmerson E, May AJ, Nathan S, Cruz-Pacheco N, Lizama CO, Maliskova L, Zovein AC, Shen Y, Muench MO, Knox SM. 2017. SOX2 regulates acinar cell development in the salivary gland. Elife. 6. doi:10.7554/eLife.26620. https://elifesciences.org/articles/26620.

Gibson DG, Young L, Chuang R-Y, Venter JC, Hutchison CA, Smith HO. 2009. Enzymatic assembly of DNA molecules up to several hundred kilobases. Nat Methods. 6(5):343-5. doi:10.1038/nmeth.1318. [accessed 2014 Jul 14]. http://www.nature.com/nmeth/journal/v6/n5/full/nmeth.1318.html.

Goomer R, Kunkel G. 1992. The transcriptional start site for a human U6 small nuclear RNA gene is dictated by a compound promoter element consisting of the PSE and the TATA box. Nucleic Acids Res. 20(18):4903-4912. doi:10.1093/nar/20.18.4903.

Graf R, Li X, Chu VT, Rajewsky K. 2019. sgRNA Sequence Motifs Blocking Efficient CRISPR/Cas9-Mediated Gene Editing. Cell Rep. 26(5):1098-1103.e3. doi:10.1016/j.celrep.2019.01.024. https://doi.org/10.1016/j.celrep.2019.01.024.

Harunaga JS, Doyle AD, Yamada KM. 2014. Local and global dynamics of the basement membrane during branching morphogenesis require protease activity and actomyosin contractility. Dev Biol. 394(2):197-205. doi:10.1016/j.ydbio.2014.08.014. 
http://dx.doi.org/10.1016/j.ydbio.2014.08.014.

Hsu JC, Koo H, Harunaga JS, Matsumoto K, Doyle AD, Yamada KM. 2013. Region-specific epithelial cell dynamics during branching morphogenesis. Dev Dyn. 242(9):1066-77. doi:10.1002/dvdy.24000. http://www.ncbi.nlm.nih.gov/pubmed/23780688.

Hsu PD, Scott DA, Weinstein JA, Ran FA, Konermann S, Agarwala V, Li Y, Fine EJ, Wu X, Shalem O, et al. 2013. DNA targeting specificity of RNA-guided Cas9 nucleases. Nat Biotechnol. 31(9):827-832. doi:10.1038/nbt.2647.

Ito S, Nagata K. 2017. Biology of Hsp47 (Serpin H1), a collagen-specific molecular chaperone. Semin Cell Dev Biol. 62:142-151. doi:10.1016/j.semcdb.2016.11.005. http://dx.doi.org/10.1016/j.semcdb.2016.11.005.

Jaskoll T, Zhou YM, Chai Y, Makarenkova HP, Collinson JM, West JD, Hajihosseini MK, Lee J, Melnick M. 2001. Embryonic submandibular gland morphogenesis: Stage-specific protein localization of FGFs, BMPs, Pax6 and Pax9 in normal mice and abnormal smg phenotypes in FgfR2-IIlc+/L, BMP7-/- and Pax6-/- mice. Cells Tissues Organs. 170(23):83-98. doi:10.1159/000046183.

Jinek M, Chylinski K, Fonfara I, Hauer M, Doudna J a, Charpentier E. 2012. A programmable dual-RNA-guided DNA endonuclease in adaptive bacterial immunity. Science (80- ). 337(6096):816-821. doi:10.1126/science.1225829. [accessed 2013 Feb 27]. http://www.ncbi.nlm.nih.gov/pubmed/22745249.

Kechagia JZ, Ivaska J, Roca-Cusachs P. 2019. Integrins as biomechanical sensors of the microenvironment. Nat Rev Mol Cell Biol. 20(8):457-473. doi:10.1038/s41580-019-01342. http://dx.doi.org/10.1038/s41580-019-0134-2.

Knosp WM, Knox SM, Lombaert IMA, Haddox CL, Patel VN, Hoffman MP. 2015.

Submandibular parasympathetic gangliogenesis requires sprouty-dependent Wnt signals from epithelial progenitors. Dev Cell. 32(6):667-677. doi:10.1016/j.devcel.2015.01.023. http://linkinghub.elsevier.com/retrieve/pii/S1534580715000660. 
Larsen M, Wei C, Yamada KM. 2006. Cell and fibronectin dynamics during branching morphogenesis. J Cell Sci. 119(Pt 16):3376-84. doi:10.1242/jcs.03079. http://www.ncbi.nlm.nih.gov/pubmed/16882689.

Lin Y, Cradick TJ, Brown MT, Deshmukh H, Ranjan P, Sarode N, Wile BM, Vertino PM, Stewart FJ, Bao G. 2014. CRISPR/Cas9 systems have off-target activity with insertions or deletions between target DNA and guide RNA sequences. Nucleic Acids Res. 42(11):7473-7485. doi:10.1093/nar/gku402.

Ma H, Wu Y, Dang Y, Choi JG, Zhang J, Wu H. 2014. Pol III promoters to express small RNAs: Delineation of transcription initiation. Mol Ther - Nucleic Acids. 3(March):e161. doi:10.1038/mtna.2014.12. http://dx.doi.org/10.1038/mtna.2014.12.

Nakao A, Inaba T, Murakami-Sekimata A, Nogawa H. 2017. Morphogenesis and Mucus Production of Epithelial Tissues of Three Major Salivary Glands of Embryonic Mouse in 3D Culture. Zoolog Sci. 34(6):475-483. doi:10.2108/zs160177. http://www.bioone.org/doi/10.2108/zs160177.

Nogawa H, Takahashi Y. 1991. Substitution for mesenchyme by basement-membrane-like substratum and epidermal growth factor in inducing branching morphogenesis of mouse salivary epithelium. Development. 112(3):855-61. http://www.ncbi.nlm.nih.gov/pubmed/1935691.

Patel VN, Rebustini IT, Hoffman MP. 2006. Salivary gland branching morphogenesis. Differentiation. 74(7):349-64. doi:10.1111/j.1432-0436.2006.00088.x. http://doi.wiley.com/10.1111/j.1432-0436.2006.00088.x.

Platt RJ, Chen S, Zhou Y, Yim MJ, Swiech L, Kempton HR, Dahlman JE, Parnas O, Eisenhaure TM, Jovanovic M, et al. 2014. CRISPR-Cas9 Knockin Mice for Genome Editing and Cancer Modeling. Cell. 159(2):440-55. doi:10.1016/j.cell.2014.09.014. http://www.ncbi.nlm.nih.gov/pubmed/25263330.

Raj A, van den Bogaard P, Rifkin S a, van Oudenaarden A, Tyagi S. 2008. Imaging individual 
mRNA molecules using multiple singly labeled probes. Nat Methods. 5(10):877-879. doi:10.1038/nmeth.1253.

Sakai T, Larsen M, Yamada KM. 2003. Fibronectin requirement in branching morphogenesis. Nature. 423(June):876-881. doi:10.1038/nature01723.1.

Schindelin J, Arganda-Carreras I, Frise E, Kaynig V, Longair M, Pietzsch T, Preibisch S, Rueden C, Saalfeld S, Schmid B, et al. 2012. Fiji: An open-source platform for biologicalimage analysis. Nat Methods. 9(7):676-82. doi:10.1038/nmeth.2019.

Sekiguchi R, Yamada KM. 2018. Basement Membranes in Development and Disease. 1st ed. Elsevier Inc. http://dx.doi.org/10.1016/bs.ctdb.2018.02.005.

Sequeira SJ, Gervais EM, Ray S, Larsen M. 2013. Genetic modification and recombination of salivary gland organ cultures. J Vis Exp.(71):1-7. doi:10.3791/50060. http://www.jove.com/video/50060/genetic-modification-recombination-salivary-glandorgan.

Steinberg Z, Myers C, Heim VM, Lathrop CA, Rebustini IT, Stewart JS, Larsen M, Hoffman MP. 2005. FGFR2b signaling regulates ex vivo submandibular gland epithelial cell proliferation and branching morphogenesis. Development. 132(6):1223-1234. doi:10.1242/dev.01690. [accessed 2016 Aug 30]. http://www.ncbi.nlm.nih.gov/pubmed/15716343.

Szymaniak AD, Mi R, McCarthy SE, Gower AC, Reynolds TL, Mingueneau M, Kukuruzinska M, Varelas X. 2017. The hippo pathway effector YAP is an essential regulator of ductal progenitor patterning in the mouse submandibular gland. Elife. 6 . doi:10.7554/eLife.23499.

Tucker AS. 2007. Salivary gland development. Semin Cell Dev Biol. 18(2):237-244. doi:10.1016/j.semcdb.2007.01.006.

Wang S. 2018. Single Molecule RNA FISH (smFISH) in Whole-Mount Mouse Embryonic Organs. Curr Protoc Cell Biol. 83(1):e79. doi:10.1002/cpcb.79. 
http://doi.wiley.com/10.1002/cpcb.79.

Wang S, Matsumoto K, Lish SR, Cartagena-Rivera AX, Yamada KM. 2021. Budding epithelial morphogenesis driven by cell-matrix versus cell-cell adhesion. Cell. 184(14):37023716.e30. doi:10.1016/j.cell.2021.05.015. https://doi.org/10.1016/j.cell.2021.05.015.

Wang S, Sekiguchi R, Daley WP, Yamada KM. 2017. Patterned cell and matrix dynamics in branching morphogenesis. J Cell Biol. 216(3):559-570. doi:10.1083/jcb.201610048. http://www.jcb.org/lookup/doi/10.1083/jcb.201610048. 


\section{A}

\section{Conventional \\ procedure}

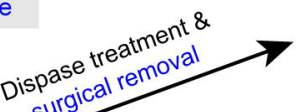
sulg

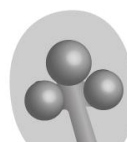

Intact mouse E13

submandibular

glands (SMGs)

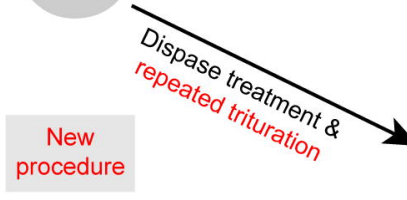

B

All + non-solidified MG; $48 \mathrm{~h}$

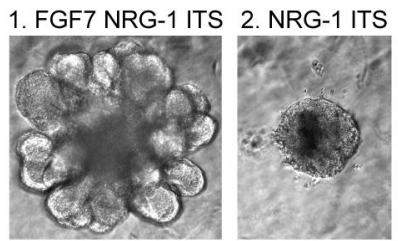

Non-solidified Matrigel in low-attachment wells

$$
\text { 3. FGF7 ITS }
$$

4. FGF7

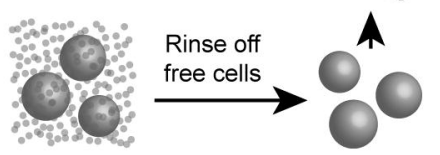

\section{C}

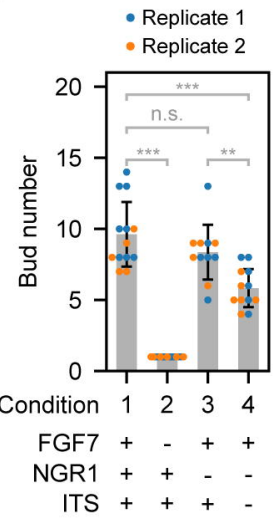




\section{A sgRNA sequence}

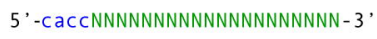
$+$

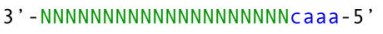

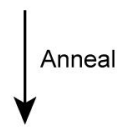

\section{sgRNA duplex}

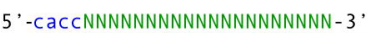
3 ' - NNNNNNNNNNNNNNNNNNNNCaaa-5,

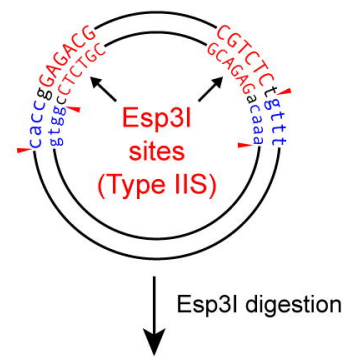

Lentiviral vector expressing sgRNA with red nuclear reporter:

\begin{tabular}{|c|c|c|c|c|}
\hline pU6 & pEF1s & NLS-mScarlet-I & BlastR & WPRE \\
\hline pU6 & pEF1s & NLS-tagBFP & BlastR & WPRE \\
\hline
\end{tabular}

T4 PNK \& T4 ligase
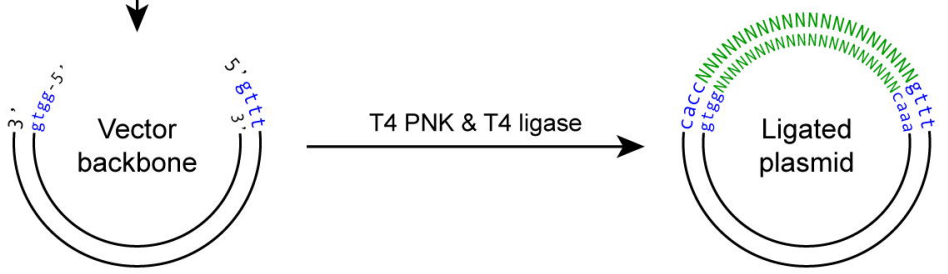

B

\begin{tabular}{|c|c|c|c|c|c|}
\hline Day 0 & Day 1 & Day 2 & Day 3 & Day 4 & Day 5 \\
\hline $\begin{array}{l}\text { Seed cells } \\
4 \times 10^{6} \\
\text { HEK293 } \\
\text { cells per } \\
10-\mathrm{cm} \text { dish }\end{array}$ & $\begin{array}{l}\text { Co-transfection } \\
\text { 1. Lenti-sgRNA } \\
\text { 2. psPAX2 } \\
\text { (packaging) } \\
\text { 3. pMD2.G } \\
\text { (envelope) }\end{array}$ & $\begin{array}{l}\text { Replace } \\
\text { media }\end{array}$ & $\begin{array}{l}\text { 1st Harvest } \\
\text { Store at } 4^{\circ} \mathrm{C} \text {, } \\
\text { replace with } \\
\text { fresh media }\end{array}$ & $\begin{array}{l}\text { 2nd Harvest } \\
\text { Pool into the } 1 \text { st } \\
\text { Add } 5 \times \text { PEG to } \\
\text { concentrate } \\
\text { Store at } 4^{\circ} \mathrm{C} \text { for } \\
\text { at least } 12 \mathrm{~h}\end{array}$ & $\begin{array}{l}\text { Collect } \\
\text { concentrated virus } \\
1500 \times \mathrm{g}, 4^{\circ} \mathrm{C}, 30 \mathrm{~min} \\
\text { Resuspend with } \\
500 \mu \mathrm{L} \text { medium }\end{array}$ \\
\hline
\end{tabular}

C

Lentivirus titration with Lenti- $X^{\mathrm{TM}}$ GoStix ${ }^{\mathrm{TM}}$ Plus

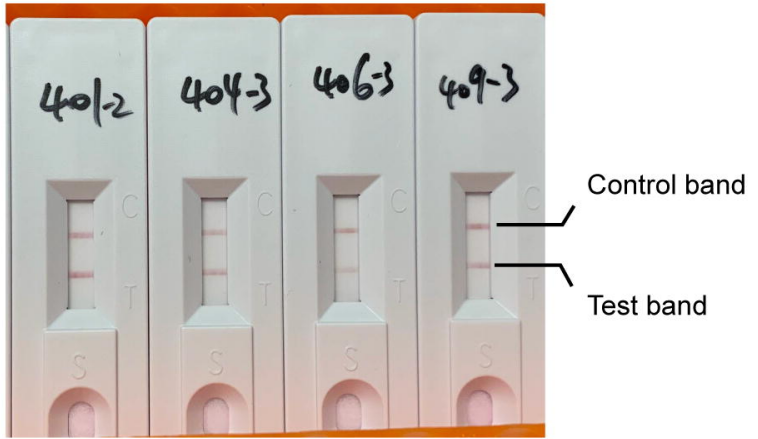

D

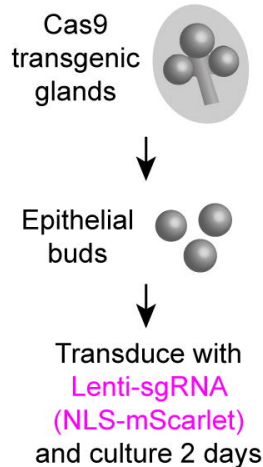


A
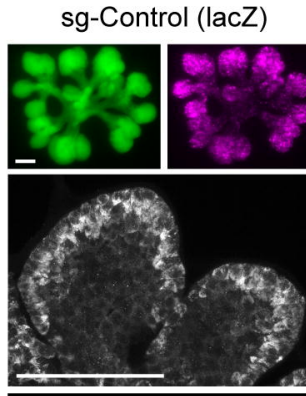

Anti-HSP47 (SERPINH1) NLS-mScarlet-I (sgRNA reporter) EGFP (Cas9 Transgene reporter) C
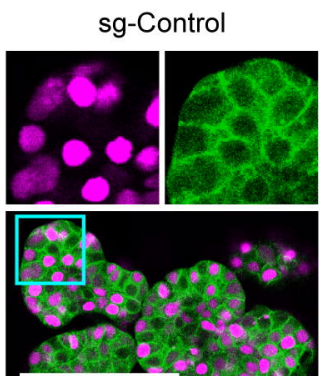

Anti-a9 integrin (ITGA9)
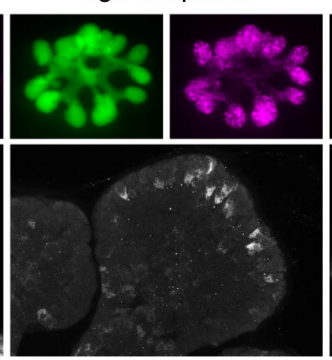

sg2-Serpinh1

sg3-Serpinh1
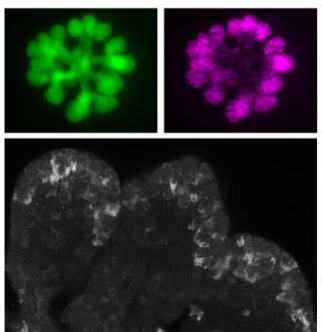

\%

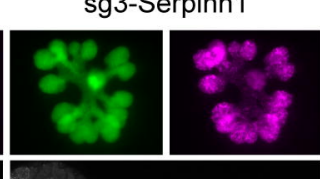

$0^{2 x} \cdot c$

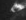
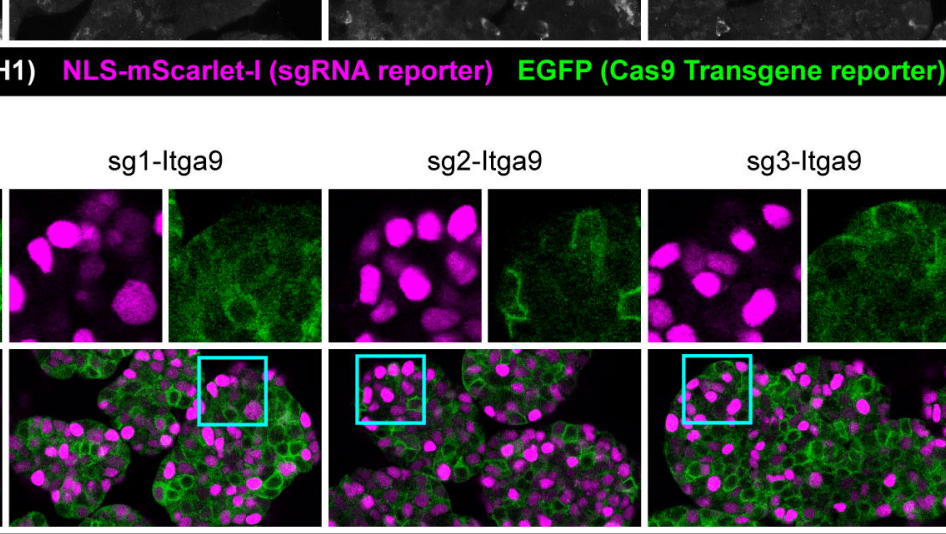

r

NLS-mScarlet-I (sgRNA reporter)
B

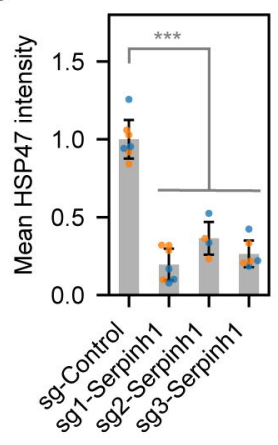

D

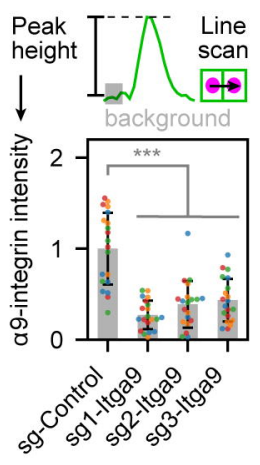




\section{A}
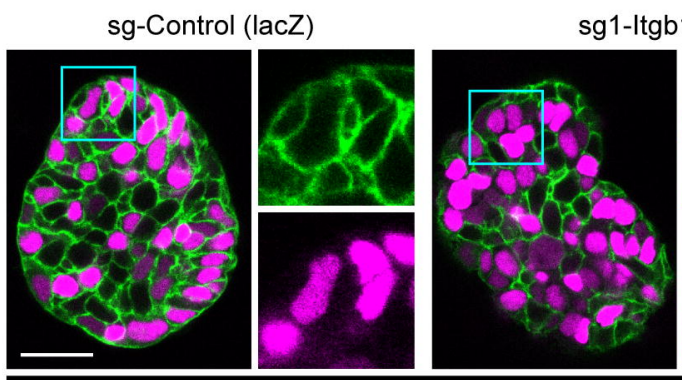

Anti- $\beta 1$ integrin (ITGB1)

C

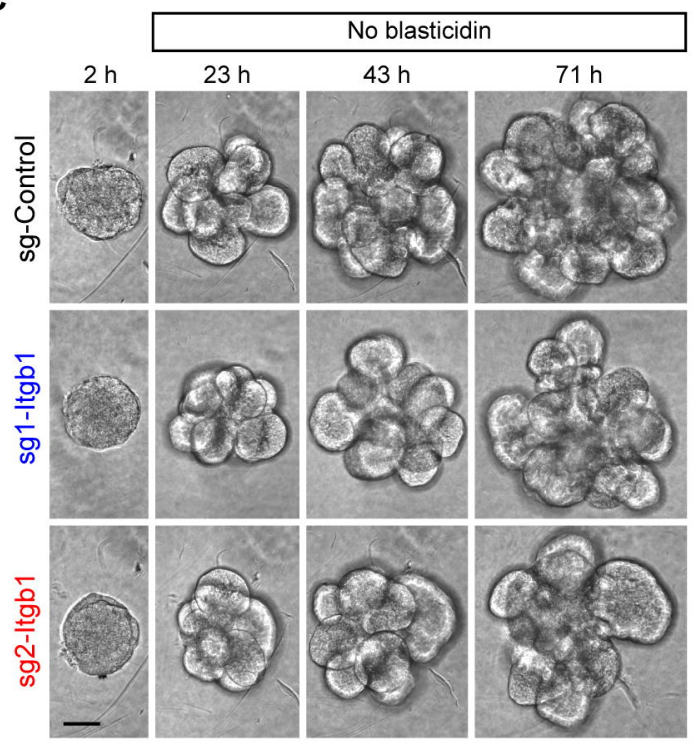

sg2-Itgb1
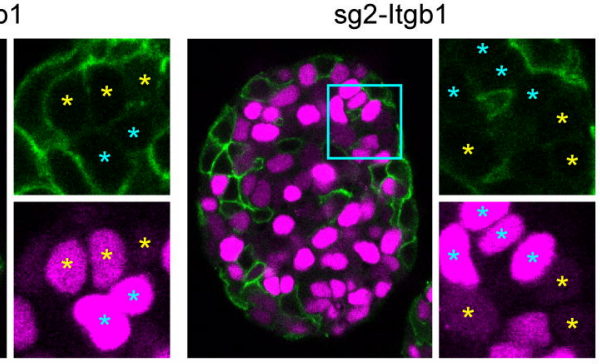

NLS-mScarlet (sgRNA reporter)

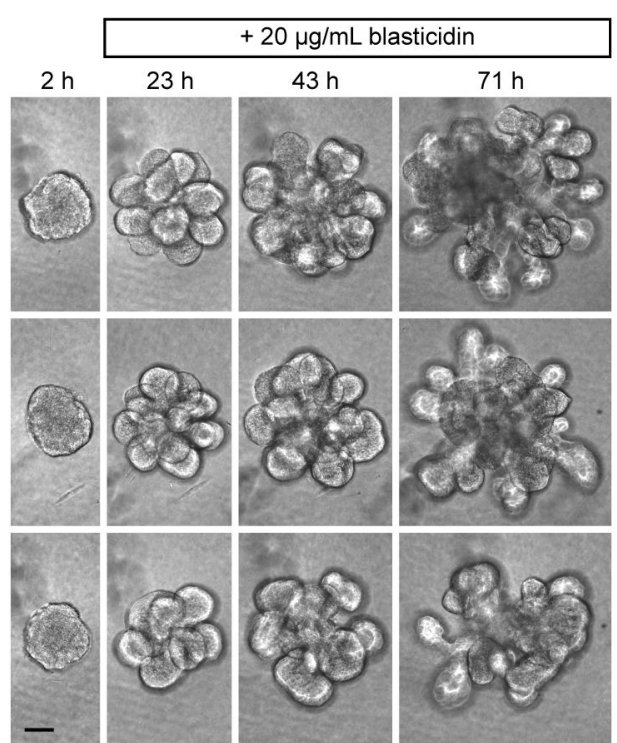

B

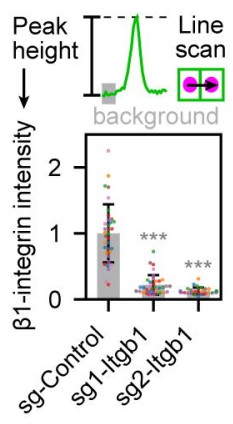

D

- sg-Control

- sg1-|tgb1

- sg2-Itgb1
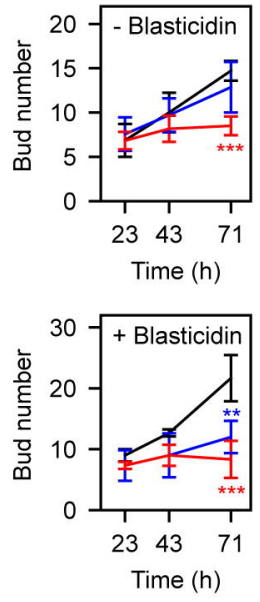